\title{
GÉRMENES PREV ALENTES EN LA UNIDAD DE CUIDADOS INTENSIVOS DE LA IPS UNIPAMPLONA DE LA CIUDAD DE SAN JOSÉ DE CÚCUTA EN EL PERIODO COMPRENDIDO ENTRE DICIEMBRE 1 DEL 2012 Y ENERO 1 DE 2015 E INFECCIONES ASOCIADAS MÁS IDENTIFICADAS.
}

\author{
PREVALENT SEEDS IN THE INTENSIVE CARE UNIT OF THE UNIPAMPLONA IPS OF THE \\ CITY OF SAN JOSÉ DE CÚCUTA IN THE PERIOD DURING DECEMBER 1, 2012 AND \\ JANUARY 1, 2015 AND MOST IDENTIFIED ASSOCIATED INFECTIONS.
}

Llerena J. Wendy J. ${ }^{1}$, Ruiz L. Jenny A ${ }^{1}$., Torres B. Bradith P. ${ }^{1}$, Algarín Holmes. ${ }^{2}$

\footnotetext{
${ }^{1}$ Grupo de investigación en Epidemiología y Salud Pública de la Universidad de Pamplona. ${ }^{2}$ Grupo de investigación en ciencias clínicas de la IPS Unipamplona
}

Recibido 21 de Julio 2016; aceptado 30 de octubre de 2016.

\section{RESUMEN}

La presente investigación tuvo como objetivo identificar los principales microorganismos en la unidad de cuidados intensivos de la IPS Unipamplona en la ciudad de San José de Cúcuta y establecer el tipo de infecciones que se presentan con mayor frecuencia dentro de esta. Este estudio se fundamentó bajo un diseño descriptivo de tipo retrospectivo a través de la recolección de datos dentro de la institución entre diciembre 1 de 2012 y enero 1 de 2015 obteniéndose una población de 484 pacientes. Se tuvo como criterios de inclusión paciente mayor de 18 años y aquellas infecciones que por su período de incubación se 
Autor a quien dirigirse la correspondencia: Wendy Johanna Llerena Jaimes. Correo electrónico: wendijaimes@gmail.com manifestaron durante la estancia del paciente en la unidad y que sean consecuencia directa de la actividad hospitalaria. Fue así como se obtuvieron los siguientes resultados: dentro de las primeros 10 gérmenes más frecuentemente aislados en la UCI de la IPS Unipamplona, la Klebsiella pneumoniae ocupo el primer lugar con un $28 \%$; el $20 \%$ de los pacientes de la unidad estaban expuestos al catéter venoso; el lavado broncoalveolar con un $51 \%$, fue el sitio en donde más se aislaron microorganismos; el antibiótico más empleado fue la Ampicilina-Sulbactam en el $24 \%$ de los casos y finalmente se encontró que la $P$. aeruginosa mostró una resistencia del $100 \%$ a antibióticos como el TrimetoprimSulfametoxazol, Tigeciclina, Ampicilina Sulbactam Cefalotina, a diferencia del $S$. aureus que presentó una mayor tasa de sensibilidad frente a diversos antibióticos.

Palabras clave: Infección intrahospitalaria, infecciones relacionadas con catéter, infecciones urinarias, neumonía asociada al ventilador.

\section{ABSTRACT}

This research aimed to identify the main microorganisms in the intensive care unit of the IPS Unipamplona in the city of San José de Cúcuta and set the type of infections that occur most often within this. This study was based under a retrospective descriptive design through data collection within the institution between December 1, 2012 and January 1, 2015 resulting in a population of 484 patients. He had inclusion criteria patients older than 18 and those infections that incubation period demonstrated during the patient's stay in the unit and are a direct result of hospital activity. It was and the following results were obtained within the first 10 germs most frequently ailsados in the ICU of the IPS Unipamplona Klebsiella 
pneumoniae ranked first with $28 \%, 20 \%$ of patients in the unit were exposed to venous catheter, bronchoalveolar lavage with $51 \%$, was the site where more microorganisms were isolated the antibiotic most often used was the Ampicillin-Sulbactam in $24 \%$ of cases and finally found that $P$. aeruginosa showed resistance $100 \%$ to antibiotics such as trimethoprimsulfamethoxazole, tigecycline, Ampicillin Sulbactam cephalothin, unlike the $S$. aureus that showed a higher rate of sensitivity to various antibiotics.

Keywords: nosocomial infection, catheter-related urinary infections, ventilator-associated pneumonia infections.

\section{INTRODUCCIÓN}

Las infecciones nosocomiales (IN) 0 denominadas actualmente como infecciones asociadas a la atención en salud (IAAS), son uno de los mayores problemas de salud pública en todo el mundo. Se consideran como el principal indicador de morbi-mortalidad en las Unidades de Cuidados Intensivos, las IAAS generan altos costos tanto sociales como económicos. Las tasas de infecciones nosocomiales varían en función de la gravedad de los enfermos, así como de la exposición a diversos factores de riesgo entre estos, los más destacados son la neumonía asociada a ventilador mecánico, la infección del torrente sanguíneo asociada a catéter venoso central, e infecciones del tracto urinario asociadas a catéter urinario que producen una alta mortalidad en los pacientes que ingresan a estas unidades. Los resultados de infecciones intrahospitalarias en $\mathrm{UCl}$ varían entre países en vías de desarrollo y países desarrollados, así tenemos que la incidencia es de 47,9 y 13,6 por ciento respectivamente (Chincha, 2012).

Aproximadamente 1,4 millones de pacientes adquieren una IAAS diariamente. El Centro para el Control de Enfermedades (CDC) estima que casi 2 millones de pacientes tendrán una infección intrahospitalaria cada año estando en un hospital de los Estados Unidos y aproximadamente 90,000 de ellos morirán por ésta causa (Esteban, 
2007). En Estados Unidos el CDC desarrolló, además, uno de los primeros programas de seguimiento y control para las enfermedades nosocomiales, llamado "Estudio sobre la Eficacia de Control de Infecciones Nosocomiales"; el establecimiento de la vigilancia se asoció a una menor tasa de incidencia de infecciones intrahospitalarias. Posteriormente, se desarrolló en este país el sistema National Nosocomial Infection Surveillance, que es un sistema voluntario y confidencial sobre el que se realizan informes mensuales de más de 300 hospitales y cuyos datos permitieron identificar los cambios en los patrones de incidencia, resistencia a antibióticos, focos, pronóstico y factores de riesgo para la infección (Barrero et al., 2016) .

En España se han desarrollado principalmente 2 sistemas de vigilancia de infecciones intrahospitalarias: el Estudio de la Prevalencia de las Infecciones Nosocomiales en España (EPINE), que es un estudio de prevalencia, realizado anualmente desde hace 24 años aproximadamente, el cual fue el primer sistema de vigilancia de este país y tiene como objetivo determinar la propensión de las tasas de las IAAS en los hospitales españoles. El Estudio Nacional de Vigilancia de Infección Nosocomial en Servicios de Medicina Intensiva (ENVIN-
UCI) desarrollado por el Grupo de Trabajo de Enfermedades Infecciosas de la Sociedad Española de Medicina Intensiva, Crítica y Unidades Coronarias desde 1994 recoge datos de las principales IAAS, asociadas a dispositivos (Blanchar, 2016).

En algunos países Latinoamericanos se vigilan las IAAS en sus instituciones pero no se cuenta con una buena estructuración en cuanto a la vigilancia epidemiológica, ni en los servicios de salud ni a nivel nacional. Pocos de estos estudios logran integrar la presencia de IAAS con la resistencia de los microorganismos y el uso de antimicrobianos (Palomar et al., 2008). En Colombia se cuenta con varios grupos de vigilancia de IAAS como: EI Grupo Para el Control de la Resistencia Antimicrobiana en Bogotá (GREBO) el cual propone una estrategia global, que permite conocer el comportamiento de la resistencia antimicrobiana en el tercer nivel de atención de los hospitales incluidos y a partir de ello, generar acciones e implementar estrategias que permitan minimizar el impacto de la misma (Safdar N, Maki DG, 2004). El centro Internacional de entrenamiento e investigación médica (CIDEIM) busca alternativas para disminuir el impacto negativo y los costos de las enfermedades infecciosas todo mediante el desarrollo de mejores estrategias diagnósticas y la 
generación de conocimiento que permita apoyar la formulación de políticas en prevención, diagnóstico, tratamiento y control de las enfermedades infecciosas en estudio (Prieto M F et al., 2008).

Finalmente, el Grupo Nacional de Vigilancia Epidemiológica de las Unidades de Cuidados Intensivos de Colombia (GRUVECO) el cual realizó en el 2007 un estudio en las $\mathrm{UCl}$ de algunos hospitales del país y determinó que la infección intrahospitalaria más frecuente era la neumonía asociada a ventilador mecánico (NAVM) $51.2 \%$, seguida de las Infecciones sintomáticas del tracto urinario (ITU) $29 \%$ y Bacteriemia asociada a catéter central $18.4 \%$ (Blanchar, 2016).

Posteriormente en un estudio realizado en el 2010 informó acerca de la distribución de los microorganismos causantes de infecciones nosocomiales asociadas a dispositivos entre Noviembre de 2007 a diciembre de 2009 determinando como los principales microorganismos emergentes a la Klebsiella pneumoniae, Pseudomonas aeruginosa, Escherichia coli, Staphylococcus aureus meticilino sensible, cándida spp. y Acinetobacter baumannii. Igualmente se mencionaron los antibióticos más usados empíricamente tendiendo en orden de frecuencia a Piperacilina / Tazobactam, Meropenem,
Vancomicina, Cefepime, Ciprofloxacina, Imipenem y Ampicilina/Sulbactam (Villalobos, 2011).

Dentro de los agentes patógenos comúnmente aislados en relación a neumonía asociada a ventilación mecánica se encuentran Streptococcus pneumoniae, Haemophilus influenzae, Moraxella catarrhalis, S.aureus y enterobacterias no resistentes (E.coli, K.pneumoniae) y otros microorganismos gram negativos multirresistentes como P.aeruginosa, Enterobacter spp, Acinetobacter baumannii complex, los cuales están implicados en el $55 \%$ a $85 \%$ de los casos y $S$. aureus implicado en el 20 a $30 \%$. En el caso de bacteremia asociada a catéter venoso central se atribuye la infección a Staphylococcus coagulasa negativo, $S$. aureus, enterobacterias y otros gérmenes como $P$. aeruginosa y Candida. En infecciones el tracto urinario los agentes causales son bacilos gram negativos E. coli, Klebsiella spp, Proteus spp, Pseudomonas spp, Serratia spp, organismos gram positivos o levaduras; alrededor del $12 \%$ son causadas por E. coli (Protocolo de vigilancia epidemiológica de las infecciones asociadas a dispositivos en unidades de cuidado intensivo, 2012). 
En estudios realizados en 20 Unidades de Cuidados Intensivos de Colombia, se observó una mortalidad de 26,9%; sin embargo, otro estudio observacional más reciente, conducido en 19 unidades de cuidados intensivos en Colombia con 1622 pacientes, se encontró una mortalidad total de 20,9 \% (Dennis, et al, 2007).

A lo largo del tiempo y después de varios estudios se ha demostrado que la administración temprana de antibióticos por lo general de amplio espectro influye a una evolución favorable del enfermo principalmente a corto plazo, mientras que a largo plazo, favorecen la aparición de flora emergente que condicionaría cambios en las resistencias a algunos gérmenes. Para llevar a cabo un tratamiento adecuado y que mejore las tasas de morbimortalidad en las unidades de cuidados intensivos se deben tener en cuenta un conjunto de estrategias y normas que mejoren y optimicen el uso de los antimicrobianos. Un plan inadecuado de antibiótico terapia predispondría la aparición de resistencias que a su vez llevarían a un aumento de la morbimortalidad y a una elevación consecuente de los costos directos (Dennis, 2007; Álvarez et al., 2000, Santana, et al., 2008). Solo cuando haya confirmación o exista una sospecha documentada o fundamentada de infección se utilizarán antibióticos, intentando distinguir de otros motivos como por ejemplo aquellos procesos inflamatorios que sean de naturaleza no infecciosa. Se ha establecido de manera general que en un tiempo aproximadamente de 48 a 72 horas una vez se haya dado la desaparición de la fiebre y de otros signos infecciosos con los que curse el paciente, se puede retirar el tratamiento antimicrobiano.

Existen casos especiales en donde el tratamiento se prolonga incluso dos semanas más, como los son manejo de pacientes inmunodeprimidos e infecciones relacionadas a bacterias multi resistentes (Pseudomonas, Acinetobacter, MRSA, o enterobacterias productoras de betalactamasas). No obstante, la persistencia de estos patógenos en la vía aérea de los enfermos con traqueotomía o ventilación mecánica prolongada en ausencia de signos infecciosos no debe ser un criterio contundente para continuación del tratamiento (Villalobos, 2011; Molina, et al., 2011; Zaragoza, et al., 2014).

Es por lo planteado anteriormente y por la ausencia de datos que documenten la implementación de estudios de vigilancia epidemiológica relacionados con las 
infecciones adquiridas en el ámbito hospitalario en las Unidades de Cuidados Intensivos a nivel regional y local, que se creyó necesario realizar un proyecto en donde se identificaran los

\section{METODOLOGÍA}

Se realiza un estudio epidemiológico descriptivo de tipo retrospectivo, en la Unidad de Cuidados Intensivos (UCl) de la IPS Unipamplona de la ciudad de Cúcuta, durante el periodo comprendido entre diciembre 1 de 2012 a enero 1 de 2015 y que incluirá a todos los pacientes hospitalizados en la unidad de cuidados intensivos en ese lapso de tiempo. En la investigación solo se incluyen aquellas infecciones que por su período de incubación se manifestaron durante la estancia del paciente en la unidad y que fueran consecuencia directa de la actividad hospitalaria (72 horas después del ingreso hospitalario del paciente) es así como se tomó como criterio de inclusión todo paciente mayor de 18 años que haya ingresado a la unidad de cuidados intensivos en el periodo comprendido entre diciembre 1 de 2012 y enero 1 de 2015.

Se tuvieron en cuenta las historias clínicas de los pacientes con un diagnóstico ya establecido, los registros del laboratorio de microorganismos más prevalentes en la Unidad de Cuidados Intensivos de la IPS Unipamplona en la ciudad de Cúcuta y así mismo establecer el tipo de infecciones que se presentan con mayor frecuencia.
Microbiología y los factores predisponentes para que se presentara la infección. Por otro lado, se excluyeron aquellos pacientes menores de 18 años, mujeres en estado de embarazo y los pacientes no infectados, o pacientes con infección adquirida en la comunidad.

Para la identificación de los principales agentes causales, factores de riesgo (sonda vesical, ventilación mecánica, catéter venoso y otros.) que generan infecciones en pacientes internados en la $\mathrm{UCl}$ de la IPS Unipamplona y el reconocimiento de los sitios de aislamiento más frecuentes de infección (secreción orotraqueal, urocultivo, absceso y hemocultivo, entre otros), se revisaron los registros de cultivos positivos tomados durante la estancia del paciente en la UCI y aportados por el laboratorio de microbiología de la Institución, además se revisaron las historias clínicas para conocer el diagnóstico clínico, los factores de riesgo a los que estuvieron expuestos 
los pacientes durante su estancia en la unidad.

La descripción del tratamiento antimicrobiano más usado para cada una de las patologías y la identificación de la sensibilidad de cada microorganismo fue posible mediante el análisis de las pruebas de sensibilidad 0 antibiogramas y los niveles de concentración inhibitoria mínima (MIC), aportados por el laboratorio de microbiología, en donde se identificó la respuesta del microorganismo a uno o varios antimicrobianos, sustentando la elección final de éste para el manejo de las infecciones en el paciente.

\section{RESULTADOS Y DISCUSIÓN}

Desde diciembre 1 de 2012 hasta enero 1 de 2015, ingresaron a la Unidad de cuidados Intensivos de la IPS Unipamplona 623 pacientes, según datos aportados por la oficina de estadística de la Institución, de los cuales se excluyeron 139 pacientes, ya fuera porque aparecían en los datos de la oficina de estadística con ingreso a la unidad, pero al momento de verificar el ingreso en el sistema de Gestión Hospitalaría de la IPS (GHIPS) éste no existía o por no cumplir con los días de estancia en la unidad requeridos para ser analizados en el estudio (mínimo tres días en la unidad).
Se calculó la tasa de mortalidad debida a procesos infecciosos en los pacientes hospitalizados en la unidad de cuidados intensivos de la IPS Unipamplona, la cual, se calculó mediante la revisión de datos aportados por la oficina de epidemiología de la IPS Unipamplona aplicando la fórmula que relaciona número de muertos durante el periodo sobre la población total por cien. Todos los datos se almacenaron y analizaron empleando el paquete estadístico SPSS 13.

Quedando una población de 484 pacientes, entre los 20 y 96 años con una media de edad de 62 años.

Tabla 1. Población- Sexos.

\begin{tabular}{|l|c|c|}
\hline \multicolumn{1}{|c|}{ SEXO } & Frecuencia & $\%$ \\
\hline Mujeres & 212 & 44 \\
\hline Hombres & 272 & 56 \\
\hline Total & 484 & 100 \\
\hline
\end{tabular}

Fuente: Autores.

Como lo indica la tabla 1 , la muestra total utilizada para el estudio fue de 484 pacientes, de los cuales 212 (44\%), corresponden al sexo femenino y los 272 
pacientes restantes (56 \%) equivalen al sexo masculino.

Como se evidencia en la tabla 2, en cuanto a la presencia de factores de riesgo de infecciones, dentro de los más prevalentes están la Ventilación mecánica con el 46.3 $\%$, catéter venoso con el $45 \%$, sonda nasogástrica con el $33.7 \%$, Traqueotomía con el $27.9 \%$ y catéter arterial con el 20.9 $\%$, observando presencia de otros factores, aunque en menor proporción.

Tabla 2. Factores de riesgo asociados a infección.

\begin{tabular}{|l|c|c|}
\hline \multicolumn{1}{|c|}{$\begin{array}{c}\text { FACTORES DE } \\
\text { RIESGO }\end{array}$} & $\begin{array}{c}\text { FRECUENCI } \\
\text { A }\end{array}$ & $\%$ \\
\hline NEFROSTOMÍA & 1 & 0,2 \\
\hline $\begin{array}{l}\text { CATETER DOBLE } \\
\mathrm{J}\end{array}$ & 1 & 0,2 \\
\hline $\begin{array}{l}\text { CATETER } \\
\text { PERITONEAL }\end{array}$ & 4 & 0,8 \\
\hline $\begin{array}{l}\text { MARCAPASO } \\
\text { TRANSVENOSO }\end{array}$ & 4 & 0,8 \\
\hline TORACOSTOMÍA & 20 & 4,1 \\
\hline SONDA VESICAL & 45 & 9,3 \\
\hline GASTROSTOMÍA & 73 & 15,1 \\
\hline $\begin{array}{l}\text { SONDA } \\
\text { OROGÁSTRICA }\end{array}$ & 76 & 15,7 \\
\hline $\begin{array}{l}\text { CATETER } \\
\text { ARTERIAL }\end{array}$ & 101 & 20,9 \\
\hline TRAQUEOSTOMÍA & 135 & 27,9 \\
\hline $\begin{array}{l}\text { SONDA } \\
\text { NASOGÁSTRICA }\end{array}$ & 163 & 33,7 \\
\hline $\begin{array}{l}\text { CATETER } \\
\text { VENOSO }\end{array}$ & 218 & 45,0 \\
\hline $\begin{array}{l}\text { VENTILACIÓN } \\
\text { MECÁNICA }\end{array}$ & 224 & 46,3 \\
\hline
\end{tabular}

Fuente: Autores.
En la figura 1., se identifican 779 muestras que fueron positivas para el aislamiento de distintos gérmenes en la Unidad de Cuidados Intensivos. Es de destacar al Lavado bronco alveolar como el tipo de muestra donde más se aislaron microorganismos en la Unidad de Cuidados Intensivos, con una frecuencia de 401 aislamientos, lo que equivale al 51 $\%$ del total de las muestras; en segundo lugar se ubica el hemocultivo con una frecuencia de 151 aislamientos positivos.

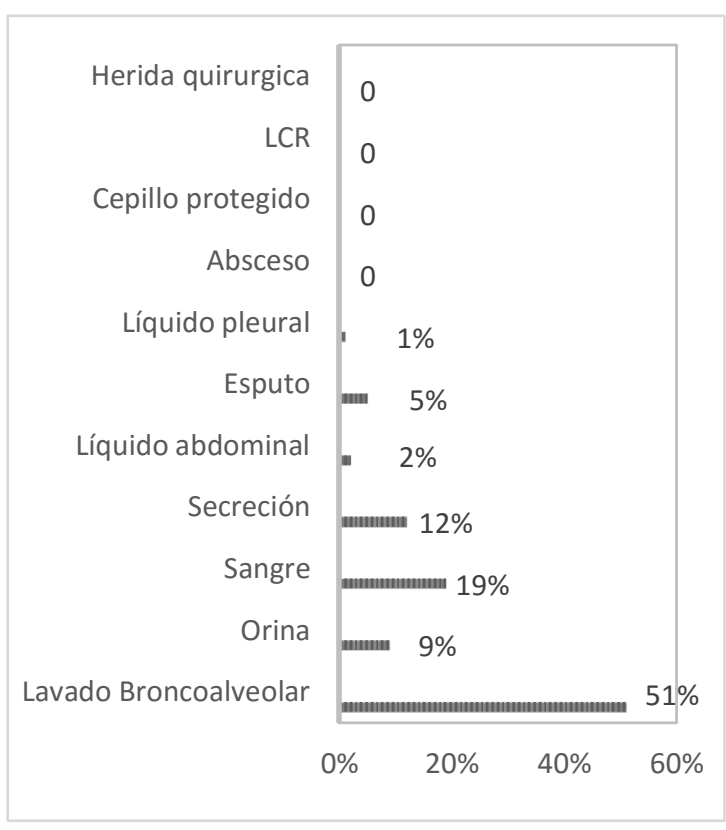

Figura 1. Principales muestras donde se aislaron microorganismos en pacientes internados en la UCI IPS Unipamplona. Fuente: Autores.

Se identificaron 804 microorganismos, en los cultivos hechos a los pacientes 
internados en la Unidad de Cuidado Intensivo de la IPS Unipamplona, teniendo como los más frecuentes: K. pneumoniae, E. coli, $P$. aeruginosa, $S$. aureus, $C$. albicans, C. Tropicalis, P. mirabilis, E. cloacae, S. Mitis, E.aerogenes, (ver figura 2).

En la figura 2 se puede observar que el microorganismo que más prevalece en la UCI de la IPS Unipamplona es la $K$. pneumoniae con un $28 \%$ del total de las muestras tomadas a todos los pacientes internados en la Unidad.

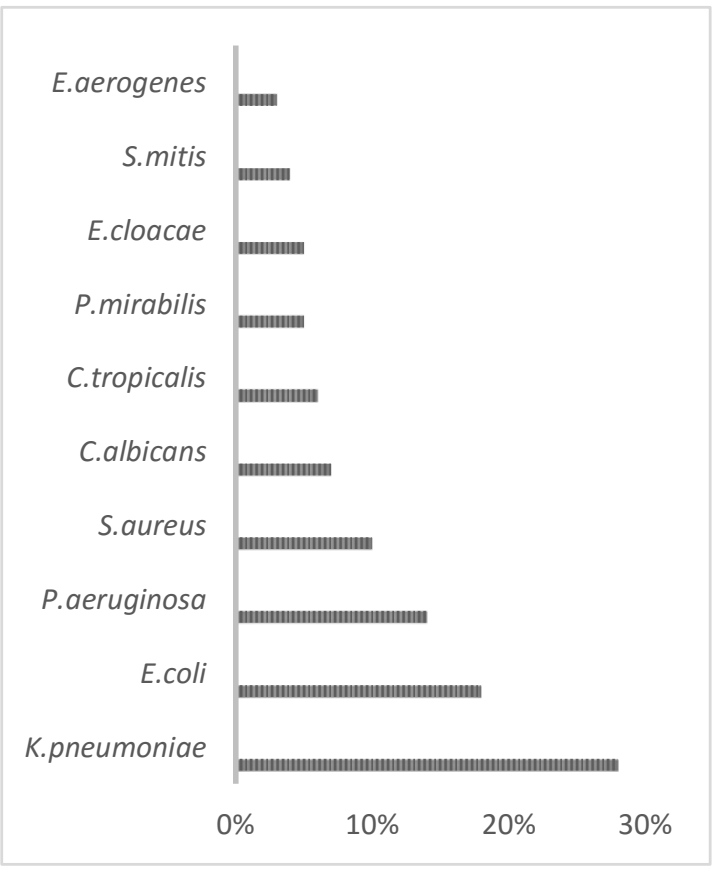

Figura 2. Microorganismos prevalentes. Fuente: Autores.

Se logra identificar además que la $P$. mirabilis y la $E$. cloacae tienen un comportamiento porcentual muy similar, siendo ambos del $5 \%$ del total de la muestra.

Como se observa en la figura 3 dentro de los 625 esquemas antibióticos más empleados en la unidad de cuidados intensivos, fue la Ampicilina - Sulbactam con una frecuencia de 152 pacientes (24 $\%$ el antibiótico más utilizado, esto independientemente del tipo de infección o germen aislado a tratar; seguido del Meropenem que tuvo una frecuencia de 96 pacientes (15\%) empleados en la unidad de cuidados intensivos.

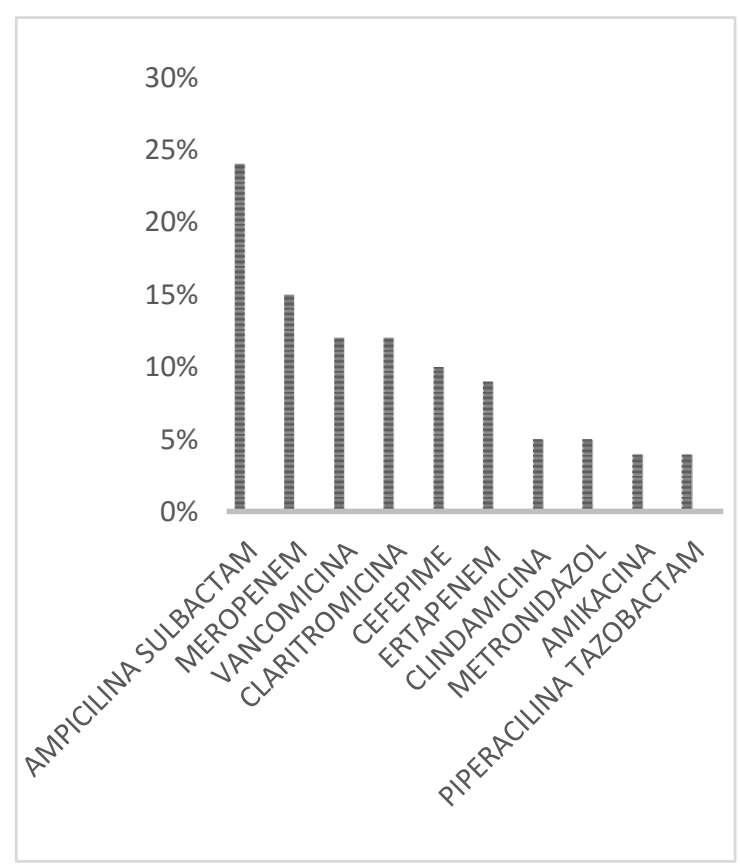

Figura 3. Relación de antibióticos empleados con mayor frecuencia en la UCI. Fuente: Autores. 
En cuanto al tiempo de tratamiento, se evidencia en la tabla 3 , que en promedio cada paciente duro con tratamiento antibiótico $10.6 \pm 10.9$ días, con rango entre 0 y 73 días. La mediana para días de tratamiento fue 7 días. Se observó relación estadísticamente significativa entre los días de tratamiento y la estancia hospitalaria $(p<0,001)$.

Tabla 3. Relación días de tratamiento-Días de estancia hospitalaria.

\begin{tabular}{|c|c|c|c|c|c|c|c|c|c|}
\hline $\begin{array}{l}\text { DIAS } \\
\text { DE }\end{array}$ & \multicolumn{8}{|c|}{$\begin{array}{l}\text { ESTANCIA HOSPITALARIA } \\
\text { (agrupado) }\end{array}$} & \multirow[t]{2}{*}{$\begin{array}{l}\text { To } \\
\text { tal }\end{array}$} \\
\hline $\begin{array}{c}\text { TRA } \\
\text { TAMI } \\
\text { ENT } \\
O\end{array}$ & $\begin{array}{c}<= \\
9\end{array}$ & $\begin{array}{c}10 \\
- \\
19\end{array}$ & $\begin{array}{l}20 \\
- \\
29\end{array}$ & $\begin{array}{l}3 \\
0 \\
- \\
3 \\
9\end{array}$ & $\begin{array}{l}4 \\
0 \\
- \\
4 \\
9 \\
\end{array}$ & $\begin{array}{l}5 \\
0 \\
- \\
5 \\
9 \\
\end{array}$ & $\begin{array}{l}6 \\
0 \\
- \\
6 \\
9\end{array}$ & $\begin{array}{l}7 \\
0 \\
+\end{array}$ & \\
\hline$<=9$ & $\begin{array}{r}21 \\
8(7 \\
9,3 \\
)\end{array}$ & $\begin{array}{r}44( \\
16, \\
0)\end{array}$ & $\begin{array}{l}\text { 9( } \\
3 \text {, } \\
3)\end{array}$ & $\begin{array}{l}2( \\
0, \\
7)\end{array}$ & $\begin{array}{l}0( \\
0, \\
0)\end{array}$ & $\begin{array}{l}\text { 1( } \\
0, \\
4)\end{array}$ & $\begin{array}{l}\text { 1( } \\
0, \\
4)\end{array}$ & $\begin{array}{l}0( \\
0, \\
0)\end{array}$ & $\begin{array}{r}275 \\
(56 \\
, 8)\end{array}$ \\
\hline $\begin{array}{c}10- \\
19\end{array}$ & $\begin{array}{r}55( \\
43, \\
0)\end{array}$ & $\begin{array}{r}54( \\
42, \\
2)\end{array}$ & $\begin{array}{l}15 \\
(1 \\
1, \\
7)\end{array}$ & $\begin{array}{l}2( \\
1, \\
6)\end{array}$ & $\begin{array}{l}1( \\
0, \\
8)\end{array}$ & $\begin{array}{l}0( \\
0, \\
0)\end{array}$ & $\begin{array}{l}0( \\
0, \\
0)\end{array}$ & $\begin{array}{l}\text { 1( } \\
0, \\
8)\end{array}$ & $\begin{array}{r}128 \\
(26 \\
, 4)\end{array}$ \\
\hline $\begin{array}{c}20- \\
29\end{array}$ & $\begin{array}{r}6(1 \\
2,8 \\
\quad \\
\end{array}$ & $\begin{array}{r}28( \\
59, \\
6) \\
\end{array}$ & $\begin{array}{r}9(1 \\
19 \\
1) \\
\end{array}$ & $\begin{array}{l}2( \\
4, \\
3)\end{array}$ & $\begin{array}{l}\text { 1( } \\
2, \\
1)\end{array}$ & $\begin{array}{l}\text { 1) } \\
2, \\
1)\end{array}$ & $\begin{array}{l}0( \\
0, \\
0)\end{array}$ & $\begin{array}{l}0( \\
0, \\
0)\end{array}$ & $\begin{array}{r}47 \\
9,7 \\
\end{array}$ \\
\hline $\begin{array}{c}30- \\
39\end{array}$ & $\begin{array}{r}3(1 \\
4,3 \\
\quad \\
\end{array}$ & $\begin{array}{r}11( \\
52, \\
4)\end{array}$ & $\begin{array}{r}5( \\
23 \\
, 8) \\
\end{array}$ & $\begin{array}{l}0( \\
0, \\
0)\end{array}$ & $\begin{array}{l}1( \\
4, \\
8)\end{array}$ & $\begin{array}{l}0( \\
0, \\
0)\end{array}$ & $\begin{array}{l}1( \\
4, \\
8)\end{array}$ & $\begin{array}{l}0( \\
0, \\
0)\end{array}$ & $\begin{array}{r}21( \\
4,3 \\
\end{array}$ \\
\hline $\begin{array}{c}40- \\
49\end{array}$ & $\begin{array}{r}0(0 \\
, 0)\end{array}$ & $\begin{array}{r}2(2 \\
5,0 \\
)\end{array}$ & $\begin{array}{r}1( \\
12 \\
, 5)\end{array}$ & $\begin{array}{r}1( \\
1 \\
2, \\
5)\end{array}$ & $\begin{array}{r}3( \\
3 \\
7, \\
5)\end{array}$ & $\begin{array}{l}0( \\
0, \\
0)\end{array}$ & $\begin{array}{r}1( \\
1 \\
2, \\
5)\end{array}$ & $\begin{array}{l}0( \\
0, \\
0)\end{array}$ & $\begin{array}{r}8(1 \\
, 7)\end{array}$ \\
\hline $\begin{array}{c}50- \\
59\end{array}$ & $\begin{array}{r}0(0 \\
, 0)\end{array}$ & $\begin{array}{r}1(2 \\
5,0 \\
)\end{array}$ & $\begin{array}{r}1( \\
25 \\
, 0)\end{array}$ & $\begin{array}{r}1( \\
2 \\
5, \\
0)\end{array}$ & $\begin{array}{l}0( \\
0, \\
0)\end{array}$ & $\begin{array}{c}1( \\
2 \\
5, \\
0)\end{array}$ & $\begin{array}{l}0( \\
0, \\
0)\end{array}$ & $\begin{array}{l}0( \\
0, \\
0)\end{array}$ & $\begin{array}{r}4(0 \\
, 8)\end{array}$ \\
\hline $60+$ & $\begin{array}{r}1(1 \\
00, \\
0)\end{array}$ & $\begin{array}{r}0(0 \\
, 0)\end{array}$ & $\begin{array}{l}0( \\
0, \\
0)\end{array}$ & $\begin{array}{l}0( \\
0, \\
0)\end{array}$ & $\begin{array}{l}0( \\
0, \\
0)\end{array}$ & $\begin{array}{l}0( \\
0, \\
0)\end{array}$ & $\begin{array}{l}0( \\
0, \\
0)\end{array}$ & $\begin{array}{l}0( \\
0, \\
0)\end{array}$ & $\begin{array}{r}1(0 \\
, 2)\end{array}$ \\
\hline Total & $\begin{array}{r}28 \\
3(5 \\
8,5 \\
) \\
\end{array}$ & $\begin{array}{r}14 \\
0(2 \\
8,9 \\
)\end{array}$ & $\begin{array}{l}40 \\
\text { (8, } \\
3)\end{array}$ & $\begin{array}{l}8( \\
1, \\
7)\end{array}$ & $\begin{array}{l}\text { 6( } \\
1, \\
2)\end{array}$ & $\begin{array}{l}3( \\
0, \\
6)\end{array}$ & $\begin{array}{l}3( \\
0, \\
6)\end{array}$ & $\begin{array}{l}1( \\
0, \\
2)\end{array}$ & $\begin{array}{r}484 \\
(10 \\
0,0 \\
)\end{array}$ \\
\hline
\end{tabular}

Fuente: Autores.

Teniendo en cuenta el tipo de microorganismo y su respuesta a los distintos antimicrobianos se puede observar que la $K$. pneumoniae presenta una resistencia del $100 \%$ para la Ampicilina, seguida de Cefalotina con un $64,3 \%$ y se observa además que para Cefixime, Cefuroxima y Cefuroxima / Acetil la resistencia es del $50 \%$. También se evidencia que la $K$. pneumoniae tiene una sensibilidad del $100 \%$ para el ácido nalidíxico y el Aztreonam, seguida de Doripenem, con un 99,3 \% y Ertapenem y Meropenem con un $97,4 \%$.

De igual forma se evidencia una sensibilidad del $100 \%$ por parte de la $E$. coli frente a la Cefixime, Doripenem y Tigeciclina. La mayor resistencia de la $\mathrm{E}$. coli fue hacia antimicrobianos como la Ampicilina, Ampicilina - Sulbactam y cefalotina con un $70.6 \%, 72.4 \%$ y $70.6 \%$ respectivamente.

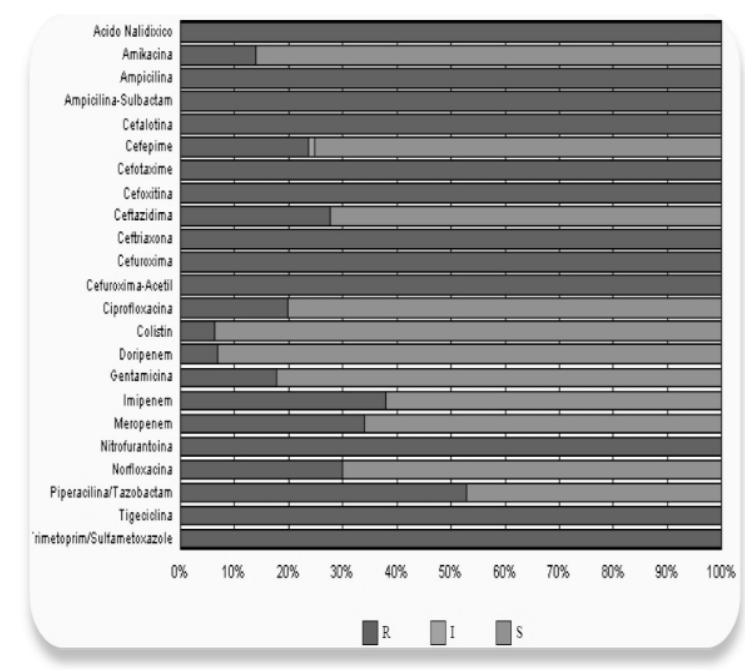

Figura. 4. Distribución total por microorganismo.

Relación

Resistencia/Sensibilidad de la $P$. 
Aeruginosa a distintos antibióticos (Tablas aportadas por el laboratorio DINAMICA de la IPS Unipamplona). [R: Resistencia, I: Intermedio, S: Sensibilidad].

En la Figura. 4 se corrobora que la $P$. Aeruginosa es un germen que presenta una alta tasa de resistencia a múltiples antimicrobianos, identificándose un $100 \%$ frente al Ácido nalidixico, Ampicilina, Ampicilina-Sulbactam, Cefalotina, Cefotaxime, Cefoxitina, Ceftriaxona, Cefuroxima, Cefuroxima-Acetil, Nitrofurantoina, Tigeciclina, TrimetoprimSulfametoxazol. Sólo se evidencia una sensibilidad cercana al $94 \%$ para Colistin y Doripenem en este microorganismo.

Con respecto al $S$. aureus es notable la buena respuesta frente a la mayoría de los antimicrobianos, contando, como se observa en la figura 5 sólo con resistencias que no superan el $50 \%$ para los Betalactámicos y Oxacilina.

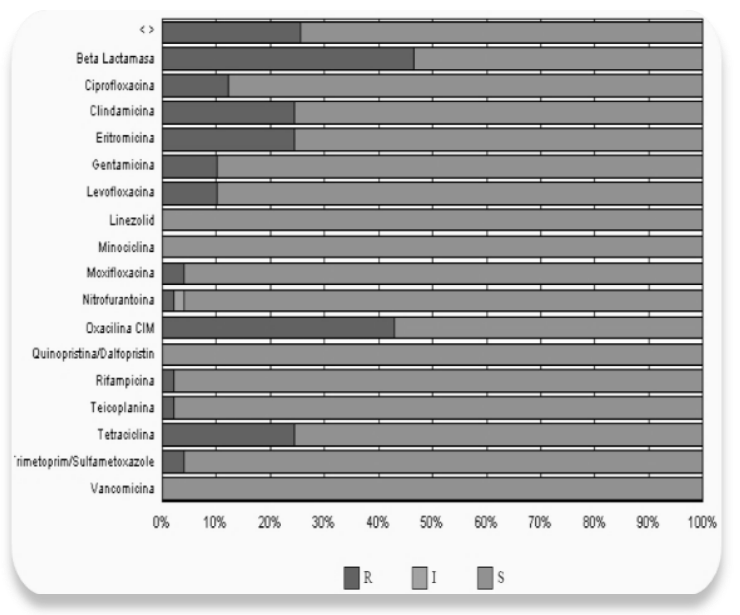

Figura. 5. Distribución total por microorganismo.

Relación Resistencia/Sensibilidad de la S. aureus a distintos antibióticos (Tablas aportadas por el laboratorio DINAMICA de la IPS Unipamplona). [R: Resistencia, I: Intermedio, S: Sensibilidad].

Como se describe en la tabla 4 el tipo de desenlace más común dentro de la Unidad de Cuidados Intensivos en la IPS Unipamplona fue el traslado a hospitalización dentro de la misma Institución, con un dato porcentual de $54 \%$ del total de la población. En segundo lugar, se ubicó el egreso por muerte con una frecuencia de 204 pacientes, es decir un $42 \%$ de la población total. Es de señalar, que solo a 5 de los pacientes (1\%) se les dio alta médica.

Tabla. 4 Tipo de egreso de la Unidad de cuidados intensivos.

\begin{tabular}{|c|c|c|}
\hline $\begin{array}{c}\text { TIPO DE } \\
\text { EGRESO }\end{array}$ & FRECUENCIA & PORCENTAJE \\
\hline Fallecidos & 204 & $47 \%$ \\
\hline $\begin{array}{c}\text { Traslado } \\
\text { interno }\end{array}$ & 262 & $48 \%$ \\
\hline $\begin{array}{c}\text { Alta } \\
\text { medica }\end{array}$ & 5 & $1 \%$ \\
\hline $\begin{array}{c}\text { Traslado } \\
\text { externo }\end{array}$ & 13 & $4 \%$ \\
\hline TOTAL & $\mathbf{4 8 4}$ & $\mathbf{1 0 0 \%}$ \\
\hline
\end{tabular}

Fuentes: Autores.

$$
\frac{\# \text { Muertos }}{\text { Población TOTAL }} \times 100
$$




$$
\frac{204}{484} \times 100=42,14 \%
$$

A partir de los pacientes fallecidos en la Unidad de Cuidados intensivos, se calculó la tasa de mortalidad aplicando la fórmula que relaciona número de muertes entre la población total por cien, dando una mortalidad de $42,14 \%$.

Tabla 5 Tipo de egreso según grupos de edad.

\begin{tabular}{|c|c|c|c|c|c|}
\hline \multirow{2}{*}{$\begin{array}{l}\text { ED } \\
A D\end{array}$} & \multicolumn{4}{|c|}{ TIPO DE EGRESO } & \multirow[t]{2}{*}{ TOTAL } \\
\hline & $\begin{array}{c}\text { FALLE } \\
\text { CE }\end{array}$ & $\begin{array}{c}\text { TRASL } \\
\text { ADO } \\
\text { INTERN } \\
0\end{array}$ & $\begin{array}{c}\text { ALTA } \\
\text { MÉDI } \\
\text { CA }\end{array}$ & $\begin{array}{l}\text { REMISIÓ } \\
\text { N A } \\
\text { OTRA } \\
\text { INSTITU } \\
\text { CIÓN }\end{array}$ & \\
\hline $\begin{array}{l}<= \\
29\end{array}$ & $\begin{array}{c}7(30,4 \\
)\end{array}$ & $\begin{array}{c}16(69, \\
6)\end{array}$ & $\begin{array}{c}0(0,0 \\
)\end{array}$ & $0(0,0)$ & $23(4,8)$ \\
\hline $\begin{array}{c}30 \\
- \\
39\end{array}$ & $\begin{array}{c}14(34, \\
1)\end{array}$ & $\begin{array}{c}25(61, \\
0)\end{array}$ & $\begin{array}{c}1(2,4 \\
)\end{array}$ & $1(2,4)$ & $41(8,5)$ \\
\hline $\begin{array}{l}40 \\
- \\
49\end{array}$ & $\begin{array}{c}16(38, \\
1)\end{array}$ & $\begin{array}{c}24(57, \\
1)\end{array}$ & $\begin{array}{c}1(2,4 \\
)\end{array}$ & $1(2,4)$ & $42(8,7)$ \\
\hline $\begin{array}{l}50 \\
- \\
59\end{array}$ & $\begin{array}{c}29(36, \\
7)\end{array}$ & $\begin{array}{c}48(60, \\
8)\end{array}$ & $\begin{array}{c}1(1,3 \\
)\end{array}$ & $1(1,3)$ & $\begin{array}{c}79(16 \\
3)\end{array}$ \\
\hline $\begin{array}{l}60 \\
- \\
69\end{array}$ & $\begin{array}{c}46(46, \\
9)\end{array}$ & $\begin{array}{c}46(46, \\
9)\end{array}$ & $\begin{array}{c}5(5,1 \\
)\end{array}$ & $1(1,0)$ & $\begin{array}{c}98(20, \\
2)\end{array}$ \\
\hline $\begin{array}{l}70 \\
- \\
79\end{array}$ & $\begin{array}{c}49(45 \\
8)\end{array}$ & $\begin{array}{c}54(50, \\
5)\end{array}$ & $\begin{array}{c}3(2,8 \\
)\end{array}$ & $1(0,9)$ & $\begin{array}{c}107(22 \\
, 1)\end{array}$ \\
\hline $\begin{array}{l}80 \\
- \\
89\end{array}$ & $\begin{array}{c}35(42, \\
2)\end{array}$ & $\begin{array}{c}46(55, \\
4)\end{array}$ & $\begin{array}{c}2(2,4 \\
)\end{array}$ & $0(0,0)$ & $\begin{array}{c}83(17, \\
1)\end{array}$ \\
\hline $90+$ & $\begin{array}{c}8(72,7 \\
)\end{array}$ & $3(27,3)$ & $\begin{array}{c}0(0,0 \\
)\end{array}$ & $0(0,0)$ & $11(2,3)$ \\
\hline $\begin{array}{l}\text { Tot } \\
\text { al }\end{array}$ & $\begin{array}{c}204(4 \\
2,1)\end{array}$ & $\begin{array}{c}262(54 \\
, 1)\end{array}$ & $\begin{array}{c}13(2, \\
7)\end{array}$ & $5(1,0)$ & $\begin{array}{c}484(10 \\
0,0)\end{array}$ \\
\hline
\end{tabular}

Fuente: Autores.

Se logró identificar asociación entre la edad y el tipo de egreso hospitalario en los pacientes, observándose que la proporción de fallecimiento aumenta con respecto a la edad.

Haciendo una revisión exhaustiva en varios artículos buscando corroborar los datos obtenidos en este trabajo se encontró un estudio realizado por Rosenthal MD et al, en el 2006 donde se identificó la ventilación mecánica como el principal factor de riesgo para generar infección con un 41 \% seguido del catéter venoso central y catéter urinario con un 30 \% y $29 \%$ respectivamente (Etella, 2008); datos que se comparan con los obtenidos en este estudio donde el principal factor de riesgo es la ventilación mecánica con el $46.3 \%$, cateter venoso con el $45 \%$ y finalmente la sonda nasogástrica con el $33.7 \%$. Podemos además comparar estos datos con los aportados por GRUVECO en año 2010 en un estudio realizado en 24 UCIS del país en donde el principal factor de riesgo encontrado fue, al igual que en esta investigación, el catéter venoso central, pero en el segundo lugar se encontraba el catéter urinario (Barrero, 2016).

Teniendo en cuenta los tipos de muestra para el aislamiento de microorganismos en los pacientes de la $\mathrm{UCI}$, se evidencio un predominio del $51 \%$ para el lavado broncoalveolar frente a los otros tipos de muestra. 
Además se puede comparar que el microorganismo más aislado en ésta muestra fue la $K$. pneumoniae a diferencia de los resultados del estudio realizado por Angel Etella et al., realizado en el hospital de SAS de Jerez, en España en el 2008 donde el microorganismo más frecuente fue el $H$. influenzae.

En los informes de ENVIN-HELICS en el cual se hace vigilancia de la infección nosocomial en Unidades de Cuidados Intensivos españolas, en el año 2012 reporto los patógenos más frecuentemente aislados ocupando el primer lugar la Pseudomonas Aeruginosa (14,5\%), seguido de Escherichia Coli (13,5\%), Enterococcus Faecalis (7,9 \%), Staphylococcus Epidermidis (5,9 \%), Klebsiella Pneumoniae (7,5\%) y, en sexto lugar, Candida Albicans (5,1 \%); en comparación con este estudio el orden de frecuencia cambia debido a que los agentes infecciosos aislado con más frecuencia fueron K.Pneumoniae (28\%), ocupando el primer lugar, seguido de $E$. Coli $(18 \%)$ que continua en el segundo lugar respecto a la información anterior, y el tercer lugar se encuentra la Pseudomona Aeruginosa (14 \%) evidenciándose una disminución de su frecuencia. En cuanto a la distribución según el tipo de antibiótico utilizado en el presente estudio, se observa que la
Ampicilina-Sulbactam fue el más utilizado con una frecuencia en 152 pacientes (24 $\%)$, seguido de Meropenem en 93 casos (15\%) y el tercer lugar compartido por Claritromicina y Vancomincina con un 12 $\%$, datos que podemos confrontar con los aportados por el "estudio de utilización de antibióticos en una unidad de cuidados intensivos de un hospital público de alta complejidad", realizado en la Universidad de Chile en el año 2013 donde se observó que la Vancomicina, Colistina e Imipenem con $46,5 \%, 46 \quad \%$ y $43,9 \quad \%$ respectivamente mostraron los mayores valores (Romo, 2013).

Algo importante de señalar es que en un estudio realizado por GRUVECO en el año 2010 señaló a la Piperacilina-Tazobactam, Meropenem y Vancomicina en ese orden de frecuencia como los antibióticos más empleados, pudiéndose así comparar con el segundo y tercer lugar dados en nuestro estudio (Blanchar, 2016).

En una investigación realizada en España en el 2006 por Frutos et al., encontraron que la mortalidad promedio en 72 Unidades de Cuidado Intensivo fue del $32,8 \%$, datos que pueden compararse con el estudio realizado por Pérez J. et al., en dos unidades de cuidados intensivos en la ciudad de Barranquilla (Colombia) en el 2008 donde la mortalidad observada fue 
de $26,9 \%$, a diferencia de estas, en el presente estudio se obtuvo una mortalidad de $42,1 \%$, significativamente más elevada que las anteriores

\section{CONCLUSIONES}

Dentro de los microrganismos más frecuentes en la UCI de la IPS Unipamplona se logró identificar la K.pneumoniae con un $28 \%$, E. coli con un $18 \%$ y finalmente P.aeruginosa con un 14 $\%$.

Al igual que en antecedentes empíricos e investigativos referenciados en el presente estudio donde se destaca la ventilación mecánica como el mayor factor de riesgo en $\mathrm{UCl}$, nuestra investigación encontró a éste como el principal factor de riesgo asociado a infecciones dentro de la Unidad.

Se reconoce al lavado broncoalveolar como el sitio más frecuente de aislamiento de gérmenes en la Unidad de cuidados intensivos de la IPS Unipamplona.

En las muestras de esputo, secreciones, liquido abdominal y lavado bronqueoalveolar el microorganismo más frecuentemente aislado fue K.pneumoniae y en las muestras de orina, y sangre $E$. coli fue el patógeno más prevalente.
La Ampicilina-Sulbactam fue el antibiótico más empleado al interior de la Unidad de Cuidados Intensivos de la IPS Unipamplona. Contrariamente la Piperacilina-Tazobactam fue el antibiótico que se utilizó con menor frecuencia en la Unidad.

En este estudio se destaca la alta tasa de resistencia del $100 \%$ generada por la Pseudomona aeruginosa frente a antibióticos como el Ácido nalidíxico, Ampicilina-Sulbactam, Ampicilina, Cefotaxime, Cefalotina, Ceftriaxona, Cefoxitina, Cefuroxima, Cefuroxima-Acetil, Nitrofurantoina, Tigeciclina y TrimetoprimSultametoxazol.en contraste el microorganismo con mayores tasas de sensibilidad fue el $S$. Aureus

En la Unidad de Cuidados Intensivos de la IPS Unipamplona, se obtuvo un elevado índice de mortalidad, que podría ser explicada por las características de la población que ingresa como es la edad avanzada, el estado clínico y las comorbilidades. 


\section{BIBLIOGRAFÍA}

Álvarez F. et al., (2005). Estudio nacional de vigilancia de infección nosocomial en unidades de cuidados intensivos. Informe del año 2000. Med Intensiva; 26(2):39-50.

Barrero G. Liliana I., et al., (2016). Protocolo de vigilancia epidemiológica de las infecciones asociadas a dispositivos en unidades de cuidado intensivo. Vigilancia y control en salud pública. Instituto Nacional de Salud pror02.0000.046. Instituto nacional de salud.

Blanchar Tania M. (2016). Protocolos de vigilancia en salud pública, vigilancia epidemiológica de infecciones intrahospitalarias, Secretaría Distrital de Salud de Bogotá dirección de salud pública.

Chincha O, Cornelio E, Valverde V, Acevedo M. (2012.). Infecciones intrahospitalarias asociadas a dispositivos invasivos en unidades de cuidados intensivos de un hospital nacional de lima, Perú. Rev Perú Med Exp Salud Pública.

Dennis R., et al., (2007). Factores asociados con la mortalidad hospitalaria en pacientes admitidos en cuidados intensivos en Colombia. Arch Bronconeumol; 38 (3):117-22.

Esteban A, Frutos-vivar F, Ferguson $\mathrm{Nd}$ et al., (2007). Sepsis incidence and outcome: contrasting the intensive care unit with the hospital ward. Crit care med; 35: 1284-9.

Etella A, Mongue I, Fontaiña L, Sainz A, Galan M. y Moreno E. (2008). Lavado broncoalveolar para el diagnóstico de neumonía en enfermos en ventilación mecánica. Unidad de cuidados intensivos Hospital del SAS de Jerez. Cadíz. España. Med Intensiva. 32:41923-Vol.32 № 9

Frutos F. et al., (2006). Utilización de la ventilación mecánica en 72 unidades de cuidados intensivos en España. Med Intensiva; 27(1):1-12.

Molina F, Díaz C, Barrera L, Dela Rosa G, Dennis R, Dueñas C, Granados M, Londoño D, Ortiz G, Rodríguez F. Y Jaimes F. (2011). Perfil microbiológico de las infecciones en unidades de cuidados intensivos de Colombia EPISEPSIS Colombia. Unidad de terapia intensiva, Clínica universitaria Bolivariana, Medellín, Antioquia. Med Intensiva Vol.35 N.2 Barcelona. 
@-LIMENTECH CIENCIA Y TECNOLOGÍA ALIMENTARIA ISSN 1692-7125. Volumen 14, No. 2, p. 5 - 22, año 2016 Facultad de Ingenierías y Arquitectura Universidad de Pamplona

Palomar M, Álvarez F, Olaechea P, Insausti J, López M J. (2008). Sociedad española de medicina intensiva y unidades coronarias. Grupo de trabajo de enfermedades infecciosas. Estudio nacional de vigilancia de infección nosocomial en servicios de medicina intensiva. ENVIC HELICS.

Pérez J, Rodríguez LC, Alcalá G. (2008). Mortalidad e infecciones nosocomiales en dos unidades de cuidados intensivos de la ciudad de Barranquilla (Colombia). Revista Científica Salud Uninorte, Vol. 24, No 1.

Prieto M F et al., (2008). Proteína C Reactiva como Factor Pronóstico de Mortalidad en la Unidad de Cuidados Intensivos. Med. Intensiva. 32(9):42430427.

Protocolo de vigilancia epidemiológica de las infecciones asociadas a dispositivos en unidades de cuidado intensivo. Vigilancia y control en salud pública pror02.0000.046. Instituto nacional de salud. 2012 - 07 -05.

Romo C. (2013). Estudio de utilización de antibióticos en una unidad de cuidados intensivos de un hospital público de alta complejidad. Facultad de ciencias químicas y farmacéuticas. Universidad de Chile. Santiago de Chile.
Safdar N, Maki DG. (2004). The pathogenesis of catheter related bloodstreams infection with noncuffed short-term central venous catheters. Intensive care med 30:62-67.

Santana L, Sánchez M, Hernández E, Eugenio P. y Villanueva A. (2008). Características y pronóstico de los pacientes mayores con estancia muy prolongada en una Unidad de Cuidados Intensivos. Med Intensiva; 32(4):15762.

Villalobos AP, Barrero LI, Rivera SM, Ovalle MV, Valera D. (2014). Vigilancia de infecciones asociadas a la atención en salud, resistencia bacteriana y consumo de antibióticos en hospitales de alta complejidad, Colombia. 2011. V Biomédica; 34 (Supl.1):67-80.

Zaragoza R, Ramírez P, López M. (2014). Infección nosocomial en las unidades de cuidados intensivos. Medicina Intensiva. Unidad de Sepsis Grave. Hospital Universitario Dr. Peset, Valencia, España. Enferm Infecc Microbiol Clin; 32(5):320-327. 